\title{
Green-Aware Routing in GMPLS Networks
}

Wang, Jiayuan; Ruepp, Sarah Renée; Fagertun, Anna Manolova; Dittmann, Lars; Ricciardi, S.; Careglio, D.

\section{Published in:}

2012 International Conference on Computing, Networking and Communications (ICNC)

Link to article, DOI:

10.1109/ICCNC.2012.6167416

Publication date:

2012

Link back to DTU Orbit

Citation (APA):

Wang, J., Ruepp, S. R., Fagertun, A. M., Dittmann, L., Ricciardi, S., \& Careglio, D. (2012). Green-Aware Routing in GMPLS Networks. In 2012 International Conference on Computing, Networking and Communications (ICNC) (pp. 227-231). IEEE. https://doi.org/10.1109/ICCNC.2012.6167416

\section{General rights}

Copyright and moral rights for the publications made accessible in the public portal are retained by the authors and/or other copyright owners and it is a condition of accessing publications that users recognise and abide by the legal requirements associated with these rights.

- Users may download and print one copy of any publication from the public portal for the purpose of private study or research.

- You may not further distribute the material or use it for any profit-making activity or commercial gain

- You may freely distribute the URL identifying the publication in the public portal

If you believe that this document breaches copyright please contact us providing details, and we will remove access to the work immediately and investigate your claim 


\section{Green-Aware Routing in GMPLS Networks}

\author{
J. Wang, S. Ruepp, A.V. Manolova, L. Dittmann \\ DTU Fotonik \\ Technical University of Denmark \\ Ørsteds P1., 2800 Kgs. Lyngby, Denmark \\ Email: $\{$ jwan, srru, anva, ladit $\} @$ fotonik.dtu.dk
}

\author{
S. Ricciardi, D. Careglio \\ UPC BarcelonaTech \\ Universitat Politècnica de Catalunya \\ C. Jordi Girona 1-3, 08034, Barcelona, Spain \\ Email: \{sergior, careglio\}@ac.upc.edu
}

\begin{abstract}
The increasing amount of traffic in the Internet has been accommodated by the exponential growth of bandwidth provided by the optical networks technologies. However, such a growth has been also accompanied by an increase in the energy consumption and the concomitant green house gases (GHG) emissions. Despite the efforts for improving energy efficiency in silicon technologies and network designs, the large energy consumption still poses challenges for the future development of Internet. In this paper, we propose an extension of the Open Shortest Path First - Traffic Engineering (OSPF-TE) protocol and a greenaware routing and wavelength assignment (RWA) algorithm for minimizing the GHG emissions by routing connection requests through green network elements (NE). The network behavior and the performance of the algorithm are analyzed through simulations under different scenarios, and results show that it is possible to reduce GHGs emissions at the expense of an increase in the path length, and, in some cases, in the blocking probability. The trade-off between emissions and performance is studied. To the authors knowledge, this is the first work that provides a detailed study of a green-aware OSPF protocol.
\end{abstract}

\section{INTRODUCTION}

The energy consumption and the concomitant green house gases (GHG) emissions of the network infrastructure are becoming a major issue in the information and communication society. The increasing transport and switching capacity required by the growing traffic demands has led to a notable raise in the energy consumption of the network elements (NE), mainly electronic routers (DXC), optical switches (OXC), reconfigurable optical add/drop multiplexers (ROADM) and signal amplifiers/3R regenerators. However, not all the NEs contribute equally to the energy consumption of the network. Routers are the most power-hungry devices, followed by the electronic signal regenerators and ROADMs, with the all optical devices such as OXC and optical amplifiers being less power consuming. Although the NEs only consume electrical energy and do not directly emit GHGs, the energy plants that feed them with energy do emit GHGs, in a quantity depending on the type of energy source exploited (e.g. oil, coal, etc.). Renewable energy sources (sun, wind, tide, etc.) are being exploited more and more, and renewable energy plants (solar panels, wind/tide turbines, etc.) are

This work was supported in part by the COST Action IC0804 on Energy Efficiency in Large Scale Distributed Systems, the Spanish Ministry of Science and Innovation under the DOMINO project (TEC2010-18522), the Catalan Government under the contract SGR 1140 and the DIUE/ESF under the grant FI-201000740. The authors also acknowledge the support received from OPNET Technologies Inc. coming alongside the traditional power grid system, offering the advantage of being green, in the sense that no GHGs are emitted during the energy production, in contrast with the dirty fossil fuel-based energy sources, like oil or coal power plants which emit large quantity of GHG during the fuel burning process. Nevertheless, not all the green energy sources are always available, since some of them are strictly related to natural phenomena such as sunlight, wind and tide. For this reason, in the modern Smart Grid infrastructure, green energy plants are placed side by side with the legacy always-available energy plants, and the power distribution grid switches from one source to the other whenever necessary, without any power interruption. Some renewable energy sources are predictable, such as the sunlight in the day-night cycle, whilst others are hard to predict, such as wind and tide, which further contributes to a dynamic energy scenario. Nevertheless, it would be profitable to know which site is currently powered by green energy sources, in order to prefer it with respect to others for minimizing the overall GHG emissions. In the context of wide area telecommunications networks (WAN), in which network nodes are spread out long distances, such a knowledge may be exploited by a properly crafted energy-aware routing algorithm that routes connections through the NEs which are currently powered by green energy sources, minimizing the overall GHG emissions of the network. Since the renewable energy sources vary with time, it is necessary to have this information updated for each node. In the modern Generalized Multiprotocol Label Switching (GMPLS) [1] controlled optical networks, such updates may be realized by Open Shortest Path First - Traffic Engineering (OSPF-TE) extensions [2]. In this paper, such an extension is presented and used to spread the energy-related information through the network on a fixed time interval. An energy-aware routing algorithm is then employed to minimize the GHG emissions of the routed connections, and the results on the network performance from a dynamic point of view are finally discussed.

\section{RELATED WORK}

A number of research works have been conducted on energy-aware routing in telecommunication networks. In [3], "power-awareness" is advocated in the design and implementation of network protocols for wire-line IP networks; the po- 
tential impact of energy-aware routing protocols is considered, but their development is left as further work. In [4] the authors propose an approach for energy saving in multilayer routing for IP/GMPLS optical networks through the implementation of sleep mode of the NEs. In [5] and [6] energy-aware RWA ILP formulations and heuristic algorithms are presented for reducing the energy consumption and the GHG emissions in WDM networks. These works assume that underlying energyaware GMPLS control plane is available to the routing algorithms. However, few works have been proposed on energyaware GMPLS extensions. In [7] a simple strategy to put links into sleep mode by re-routing lightpaths is proposed and an extension of the link state advertisement (LSA) of OSPF is described to specify whether a link is in sleep mode, so that nodes avoid the sleeping links for routing lightpaths. On the same topic, in the IETF Internet draft [8] a work in progress is presented for extending the GMPLS protocols to support energy efficient traffic engineering features by adding new link/LSP status and link power on/off capability, aiming at distinguishing between down and sleeping links. Nevertheless, none of the previous works takes into consideration the type of energy plants that are currently feeding the NEs, differentiating between green and dirty sources of energy, with a dynamic performance study.

\section{GREen-AwARe Routing Design}

Routing in optical networks is different from routing in IP networks, provided that various physical constraints (e.g. wavelength availability, wavelength converters, regenerators, etc.) are taken into consideration. Optical switches consume different amount of power in processing various types of traffic, namely transparent pass-through traffic and opaque traffic. Thus, routing through alternative routes in optical networks can result in the use of different number of regenerators, wavelength converters, and of course, different number of hops. Employing all the energy-related concepts in the routing decision process would result in a complex energy model, also taking into account the trade-offs between network resource usage and power savings. As a first step towards a comprehensive energy-aware routing design, the paper focuses on a green-aware routing algorithm that uses the information provided by an extended OSPF-TE protocol to reduce the GHG emissions. A simple energy model is developed, the required OSPF-TE extensions are defined and the performance of the proposed routing algorithm is evaluated in a real mesh network topology under different simulation scenarios.

\section{A. The energy model}

In order to evaluate the possibilities offered by the proposed green-aware routing protocol, green and dirty sources of energy are differentiated at the control plane level. All the traffic is assumed to be of the same type and no wavelength conversions or regenerations are considered. The network is modeled as an undirected graph $G=(V, E)$, with the nodes $V$ being the OXCs and the edges $E$ being the optical links. Both nodes and links can be powered by green or dirty energy source, and the energy sources availability is updated on prefixed time basis. Green energy sources represent energy from wind mills, solar panels or hydro-electrical plants, whilst dirty energy sources models energy from coal, fuel or gas. The proposed model defines two types of cost value: the values 0 and 1 represent green and dirty energy source respectively. In the proposed model, both link and node energy costs influence the routing decision. However, since the Dijkstra algorithm only considers edges weights in the path computation, the costs assigned to the edges should be properly revised to include the node energy type as well. Thus, the value of the edges cost is calculated according to equation (1):

$$
c_{i j}=L_{i j}+N_{i}+N_{j},
$$

where $c_{i j}$ is the energy cost per edge, $L_{i j}$ represents the energy cost on link $(i, j)$ and $N_{i}$ represents the energy cost on node $i$. Note that equation (1) correctly sums two times the energy costs of intermediate nodes in the path $P$ (since they send and receive traffic), and only one time the energy costs of source and destination nodes (since they just send or receive traffic). The cost $C_{s d}$ of path $P$ from source $s$ to destination $d$ is the sum of all the NEs costs in the $P$ :

$$
C_{s d}=\sum_{(i, j) \in P_{E}} c_{i j},
$$

where $P_{E}$ is the set of edges of path $P$. In this way, both node and link energy costs are taken into consideration.

\section{B. Routing algorithm}

In dealing with dynamic routing requests, Open Shortest Path First - Traffic Engineering (OSPF-TE) [9] is used as the routing protocol, and Resource Reservation Protocol - Traffic Engineering (RSVP-TE) [10] as the signaling protocol. The energy-aware routing algorithm exploits information carried by the TE LSAs [9] to optimize the routing of the connections according to the proposed energy model. In order to enable energy-awareness into the routing process, the existing OSPFTE protocol is extended to spread energy related information between nodes, so that it can be used in the routing decision. The extension is made by adding a new Type, Length, Value (TLVs) to the TE extension for OSPF-TE, which carries the energy source type information. Accordingly, to achieve the goal of green-aware routing, the routing decision is based on the "lowest GHG emissions", rather than on the "shortest path" or, equivalently, on the "shortest-cost path", where the costs are the ones given by our energy model.

According to the information needed for the routing calculation, two types of energy related LSAs are flooded, node energy cost and link energy cost. Node energy cost LSAs are only flooded to their direct neighbors, since the node energy cost information is only needed when calculating the edge energy cost. Upon calculating edge energy cost, the information is flooded by link energy cost LSA over the whole network area. The routing calculation is based on a standard Dijkstra algorithm used in the OSPF-TE protocol. The "cost" 


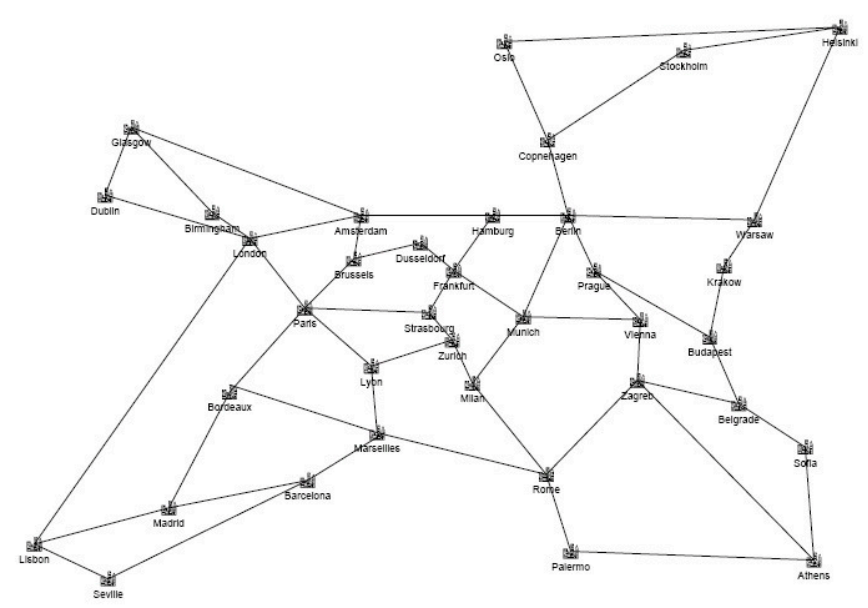

Fig. 1. Network topology used in simulations [12].

assigned in route calculation is updated by the energy cost carried in TE LSAs after a fixed time interval. According to the definition of sub-TLVs for TE LSA, the defined sub-TLVs may occur at most once in each TE LSA.

\section{Simulation Scenarios AND Results}

The energy model and the proposed OSPF-TE extension are implemented in the event driven simulator OPNET [11]. The test network topology used in the simulations is shown in Figure 1, with thirty-seven nodes and fifty-five bidirectional links, each with sixty-four wavelengths. No wavelength converters or regenerators are used. LSP connection requests are generated as a Poisson process, with exponentially distributed connection duration. The total traffic load is given by the connection duration and inter-arrival rate, and is maintained at a fixed value of 12 Erlangs. In the simulation model, first fit algorithm is applied for the wavelength assignment. We chose such a network topology to simulate a real autonomous system scenario within a single OSPF domain.

At fixed time intervals, a new update of the power source information is originated and flooded between neighbors using the proposed OSPF-TE extension. This fixed time interval is referred to as PowerChangeTime in this paper. Upon flooding the TE LSA over the whole network, the new energy cost is updated in each LSA database, and thus, used for routing calculation. In the simulation model, considering the number of nodes involved in the flooding process, no designated routers [9] for OSPF-TE protocol are used, i.e. LSAs are flooded in a broadcasting manner.

Three performance metrics have been evaluated: Energy cost (Figure 2 and Figure 3), path length (in hop count) (Figure 4 and Figure 5), connection blocking probability $(\mathrm{Pb})$ (Figure 6 and Figure 7). Results of our proposed model are discussed and evaluated, with focus on the energy cost reduction. As a comparison with the proposed energy efficient (EE) routing algorithm, results using a hop based shortest path (SP) algorithm are presented as well. The simulation parameters under

\begin{tabular}{c||c|c|c} 
& $\begin{array}{c}\text { Connection } \\
\text { Duration }\end{array}$ & $\begin{array}{c}\text { Mean Interarrival } \\
\text { Time }\end{array}$ & PowerChangeTime \\
\hline \hline Scenario 1 & 6 hours & $30 \mathrm{~min}$ & $1 / 6 / 12 / 18 / 24$ hours \\
\hline Scenario 2 & 12 hours & $60 \mathrm{~min}$ & $1 / 6 / 12 / 18 / 24$ hours
\end{tabular}

TABLE I

SIMULATION PARAMETERS.

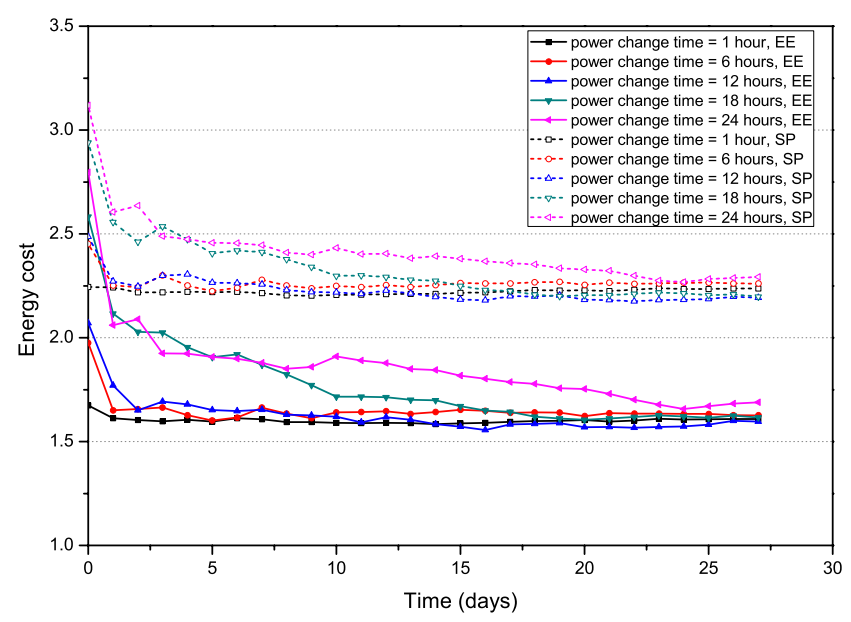

Fig. 2. Energy cost of routes under scenario 1 .

the two different investigated scenarios are shown in Table I. The offered traffic load in both scenarios is 12 Erlangs with different connection duration and mean interarrival time. Five different update intervals (PowerChangeTime) are evaluated from a value of 1 to 24 hours, with steps of 6 hours, during a four weeks simulation runtime.

\section{A. Energy cost}

In Figure 2, the energy cost of the SP and the proposed energy efficient (EE) routing algorithms are shown. As it can be seen, whatever the power change times, lower energy costs are achieved by the EE algorithm, compared to the shortest path (SP) algorithm. The energy cost using the EE algorithm is $26 \%$ to $27 \%$ lower compared to the energy cost using the SP algorithm, and the changes of PowerChangeTime interval do not have impact on this energy cost difference between the two algorithms. By increasing the connection duration from 6 hours (scenario 1) to 12 hours (scenario 2), while keeping the same offered traffic for both scenarios, a similar result is achieved. From the results in can be seen that both routing algorithms are insensitive to the PowerChangeTime with respect to the obtained energy cost.

\section{B. Routing hops}

Figure 4 and Figure 5 show the average path length (in hop count) obtained by the two algorithms for both considered scenarios. The EE algorithm reduces the energy costs by routing the connections preferring green NEs, i.e. avoiding dirty NEs, which in turns is reflected as an increase in the mean number of hops with respect to the SP algorithm. 


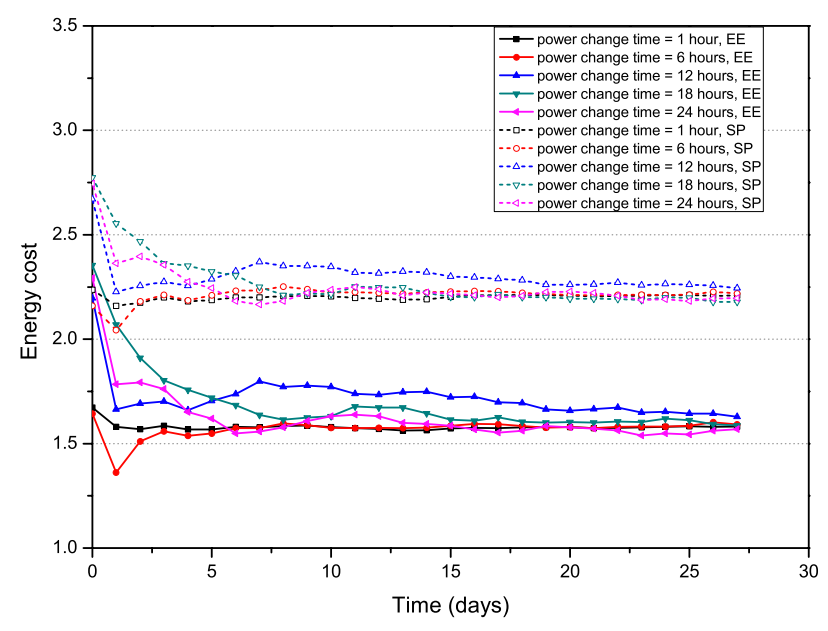

Fig. 3. Energy cost of routes under scenario 2.

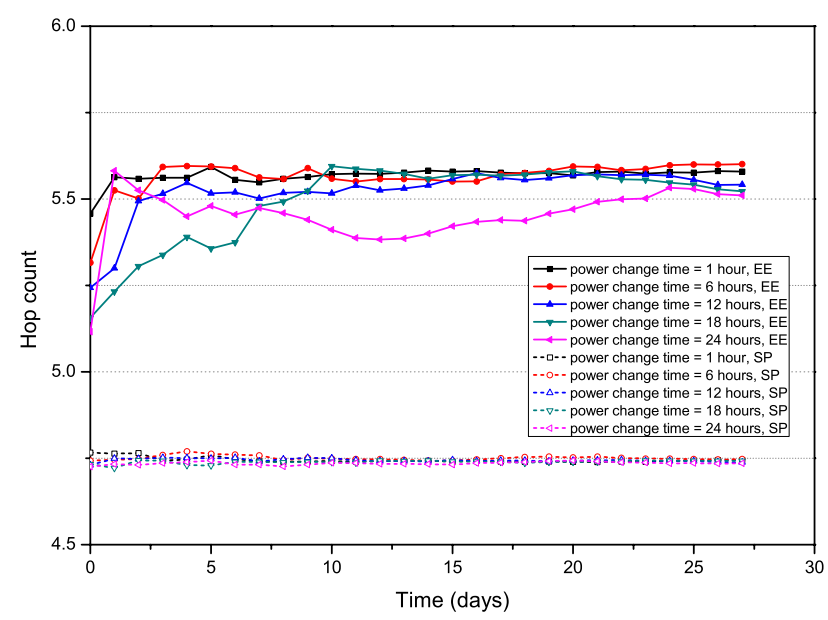

Fig. 4. Number of hops under scenario 1.

The average hop count increases from 4.75 (obtained by the SP algorithm) to 5.60 hops (by the $\mathrm{EE}$ algorithm), i.e. almost one hop more on average for both the simulation scenarios. Considering that the test network has a diameter of 36 hops, such an increase in the path length is marginal. In both scenarios neither PowerChangeTime interval nor the connection duration influences the results under SP algorithm, which is as expected. The variations in the results under the EE algorithm do not show a direct impact from changing the PowerChangeTime interval either. Based on the results of the energy cost and the path length, it is seen that the proposed EE algorithm has the ability to route the traffic away from dirty sources, thus reducing GHG emissions while keeping the hop count in an acceptable range for the considered network.

\section{Connection blocking probability}

The sensitiveness of the algorithm to the connection blocking probability $(\mathrm{Pb})$ was analyzed, and the results are plotted

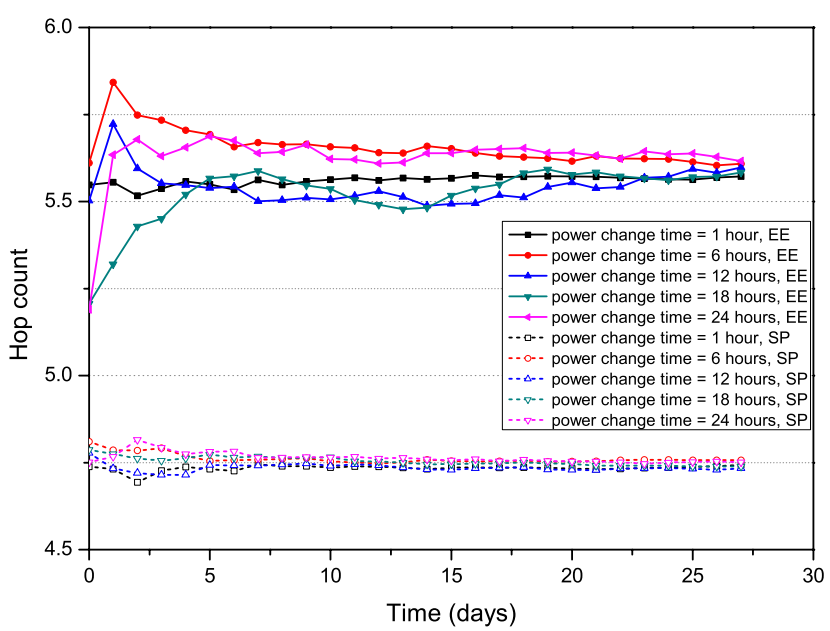

Fig. 5. Number of hops under scenario 2.

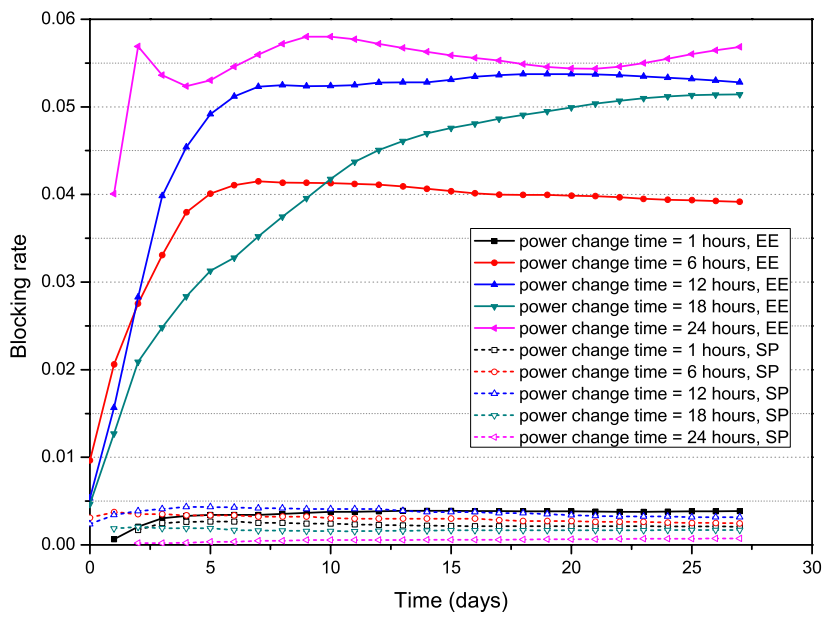

Fig. 6. Connection blocking probability under scenario 1 .

in Figure 6 and Figure 7. Under the SP algorithm, the $\mathrm{Pb}$ rates is less than $0.3 \%$ for both considered scenarios: since no energy-related information is taken into account by the SP algorithm, the PowerChangeTime interval does not influence its $\mathrm{Pb}$ rates.

The behavior of the EE algorithm is twofold. When the PowerChangeTime interval is long (6 to 24 hours), the $\mathrm{Pb}$ rates of the proposed $\mathrm{EE}$ algorithm increase as consequence of the higher mean hop count, which consumes an higher number of resources for avoiding dirty NEs. This situation is further stressed by the fact that between two consecutive power change intervals, more and more requests are routed towards the greener power sources. Certain "hot-pot" effect is created along the route with higher number of green energy sources, and the trend does not change until next power change time update arrives, which results in more blocked requests. Thus, the longer the PowerChangeTime interval is, the more severe the "hot-pot" effect is, and the higher the blocking 


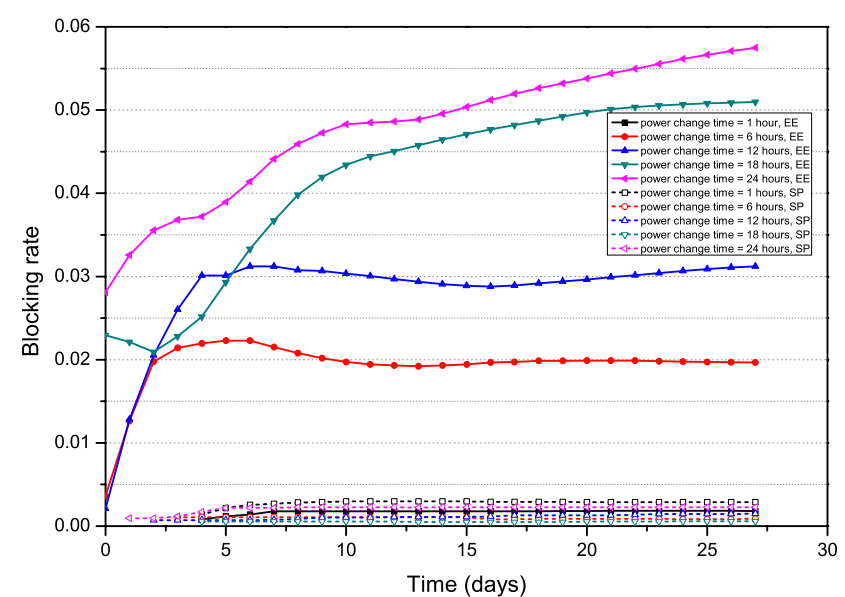

Fig. 7. Connection blocking probability under scenario 2 .

rate will be. Observing the difference between Figure 6 and Figure 7, it can be seen that a lower $\mathrm{Pb}$ rate is obtained under scenario 2. It is due to the smaller number of requests compared to requests under scenario 1 , which also means that less requests are routed and contribute to create the "hot-pot" effect. For both scenarios, the increase in the $\mathrm{Pb}$ may reach peaks of $6 \%$, which may be unacceptable for a commercial network. Although the proposed EE algorithm results in an increased blocking probability, a well-designed PowerChangeTime interval can keep the blocking probability at an acceptable rate. In the proposed simulation environment, when the PowerChangeTime interval is short (1 hour), the $\mathrm{Pb}$ rates of the EE algorithm decreases dramatically, settling down at values as low as $0.3 / 0.4 \%$ under scenario 1 , and $0.2 \%$ under scenario 2, i.e. comparable or even lower than the SP. This is due to the "balancing effect" of the energy costs on links that counterbalance the typical congestion on the overloaded links of the SP algorithm. The frequent changes in the green energy source availability forces the EE algorithm to spread the connections over the whole network, favoring better network load balancing and, thus, lower blocking probability. However, in the simulated environment, lowering the PowerChangeTime interval to achieve a lower blocking rate is possible as long as the PowerChangeTime parameter is adjustable. In the real world, the adjusting of the parameter also depends on the availability of the renewable energy sources, which might in turn make the parameter less adjustable. For example, an area of nodes where green sources are always available, will result in updates with the same value. It should also be noted that the need for a more frequent flooding of proposed EE LSA, will also create more overhead in the network, prolonging the network convergence time. A trade-off for choosing the correct update frequency, taking the network convergence time and overhead into consideration, can be further investigated.

\section{CONCLUSions And Future Work}

This paper proposes a green-aware routing algorithm through proper OSPF-TE protocol extensions. Greenawareness is enabled by flooding energy source information over the network, which is used in OSPF-TE routing decisions to lower the GHG emissions. Observing the behavior of the proposed algorithm under different scenarios, it is seen that the proposed algorithm can save up to $27 \%$ of the GHG emissions (in terms of cost unit) at the expense of a marginal increase in the path length, compared to traditional shortest path routing algorithm. As a consequence of the higher mean path length and of the created "hot pot" effect, the blocking probability may increase up to $6 \%$ when the energy source updating interval is long. On the contrary, when the interval is short, the blocking probability of the EE algorithm reaches values even better than the traditional SP algorithm, due to the better load balancing induced by its energy cost function. However, this option may be limited due to the real dynamics of the green and dirty sources. An operator should also consider extra network overhead, and the possible additional expenses for obtaining the information from Smart Grid network.

As a first step in designing an energy efficient optical network, the proposed approach gives a direct insight into the behavior of a green-aware routing algorithm. Further studies are focused on building a more realistic energy model, with more network elements taken into account each characterized by its own power consumption, that will allow to quantify the actual GHG emissions and savings. A more complex routing schema can be used, utilizing advanced constraint-based RWA algorithms. Also, the extra network overhead for spreading the energy information as soon as a change in the energy source occurs can be studied, and the trade-off between the update frequency and the performance may be further investigated.

\section{REFERENCES}

[1] E. Mannie (ed.), "Generalized Multi-Protocol Label Switching (GMPLS) Architecture," RFC 3945, Oct. 2004.

[2] D. Katz, "Traffic Engineering (TE) Extensions to OSPF Version 2," RFC 2370, Sep. 2003.

[3] J. Chabarek, J. Sommers, P. Barford, "Power Awareness in Network Design and Routing," IEEE INFOCOM, 2008.

[4] M. Nazri, M. Warip, Ivan Glesk, Ivan Andonovic, "GMPLS Energy Efficiency Scheme for Green Photonic Networks," in Proc. ICTON 2010, 2010.

[5] S. Ricciardi, D. Careglio, F. Palmieri, U. Fiore, G. Santos-Boada, J. Solé-Pareta, "Energy-Aware RWA for WDM Networks with Dual Power Sources," in Proc. IEEE ICC 2011, Kyoto, Japan, Jun. 5-9 2011.

[6] L. Chiaraviglio, M. Mellia, F. Neri, "Energy-aware Backbone Networks: a Case Study," in Proc. GreenComm'09, Dresden, Germany, June 2009.

[7] I. Cerutti, N. Sambo, P. Castoldi, "Distributed Support of Link Sleep Mode for Energy Efficient GMPLS Networks," in Proc. ECOC 2010, 19-23 September, Torino, Italy, 2010.

[8] S. Okamoto (ed.), "Requirements of GMPLS Extensions for Energy Efficient Traffic Engineering," IETF Internet-Draft, Jun. 2011.

[9] J. Moy, “OSPF Version 2," RFC 2178, Apr. 1998.

[10] L. Berger (ed.), "GMPLS Signaling Resource ReserVation ProtocolTraffic Engineering (RSVP-TE) Extensions," RFC 3473, Jan. 2003.

[11] OPNET Technologies, Inc., http://www.opnet.com.

[12] M. L. R. Inkret and B. Mikac, "WDM Network Availability Performance Analysis for the COST 266 Case Study Topologies," available on the internet, 2003. 